\title{
Stem cell-derived exosomes and the failing heart: Small cause, big effect
}

\author{
Philippe Menasché, MD, PhD
}

\footnotetext{
From the Department of Cardiovascular Surgery, Université Paris Descartes, Sorbonne Paris Cité, INSERM U-970, Hôpital Européen Georges Pompidou, Paris, France.

Received for publication April 2, 2018; accepted for publication April 5, 2018; available ahead of print May 18, 2018.

Address for reprints: Philippe Menasché, MD, PhD, Department of Cardiovascular Surgery, Hôpital Européen Georges Pompidou, 20 Rue Leblanc, 75015 Paris, France (E-mail: philippe.menasche@aphp.fr).

J Thorac Cardiovasc Surg 2018;156:1089-92

$0022-5223 / \$ 36.00$

Copyright (C) 2018 by The American Association for Thoracic Surgery

https://doi.org/10.1016/j.jtcvs.2018.04.018
}

Feature Editor's Note-During the past 2 decades, research into the use of stem cells to regenerate damaged myocardium has failed to demonstrate therapeutic efficacy commensurate with the expectations of the therapy. More recent investigations, however, have discovered a subset of small extracellular vesicles, termed exosomes, secreted by stem cells, that confer cardioprotective and regenerative effects similar to their cells of origin. Exosomes are formed through invaginations of endosomes, forming multivesicular bodies in the cytoplasm, and contain abundant amounts of RNAs, lipids, and proteins, reflecting in part the composition of the parent cell. Paracrine signaling, thought to be the major explanation for the benefit of stem cell therapy, is further extended by the concept of exosomes being a major player in cell-to-cell as well as organ-to-organ communication. Exosomes obtained from an appropriate regenerative cell source could have important beneficial effects in the regenerative processes of damaged myocardium and represent a new potential therapeutic agent. In this issue of the Journal, Menasche provides an insightful commentary on the state of the field of research of exosomes and provides important perspective and counterpoints to the recent commentary by Yang published in the March 2018 issue of Circulation Research.

\section{Francis Pagani, MD}

In the Viewpoints section of the March 2018 issue of Circulation Research, Yang $^{1}$ highlights the potential interest of exosomes in precision medicine for treating heart failure.

Exosomes belong to a heterogeneous group of nanoscale or microscale extracellular vesicles secreted by all cells in the body (along with microparticles and apoptotic bodies) and differing in their biogenesis, content, and functions. ${ }^{2,3}$ The growing interest in exosomes, the smallest of these particles (40-150 nm), stems from their ability, wellknown for years by oncologists, ${ }^{4}$ to transfer their cargo

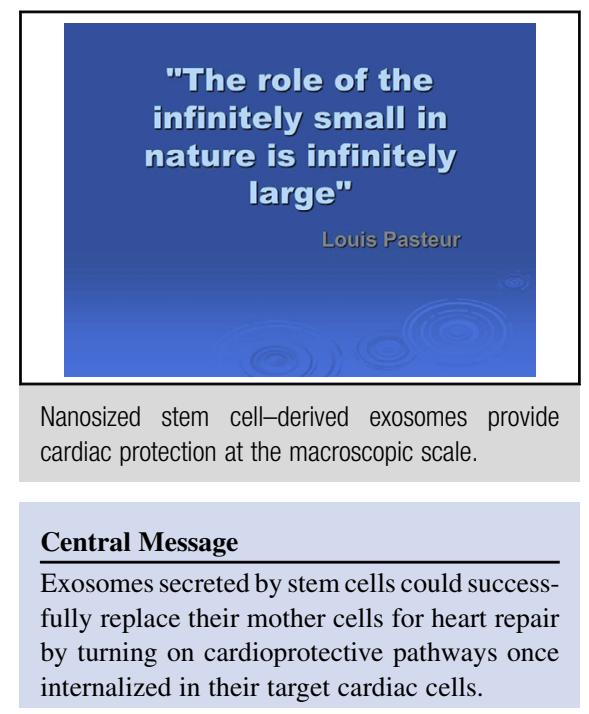

into the recipient cells. ${ }^{5}$ This cargo is particularly rich in proteins and noncoding nucleic acids (messenger RNAs, microRNAs [miRNAs]), and by shuttling it into the target cells, exosomes can profoundly modulate the activity of key signaling pathways, leading to marked changes in function. This is well illustrated by the observation that coincubation of fibroblasts with cardiosphere-derived exosomes renders the former less profibrotic while endowing them with an angiogenic potential. ${ }^{6}$ During recent years, the growing recognition that transplanted stem cells engraft in the myocardium only transiently, even though their beneficial effects may be sustained with time, has led to privileging a paracrine mechanism of action whereby the grafted cells secrete a medley of factors that harness endogenous repair pathways. For a long period, these factors have been poorly characterized; the recognition that many of them are clustered in exosomes now leads to the hypothesis that these particles might be key mediators of the paracrine effects of the transplanted cells. ${ }^{7}$ Indeed, in several preclinical models of various diseases, exosomes have been shown to duplicate faithfully the protective effects of their parent cells. ${ }^{8}$ and their benefits extend to reperfused myocardial infarction ${ }^{9-11}$ and chronic postinfarct left ventricular dysfunction. ${ }^{12-14}$ Put together, these data have raised the possibility that exosomes might successfully substitute for their parent cells. Such a switch could have substantial advantages in terms of practicality and thus clinical usefulness, such as the possibilities of large-scale production, stability after cryostorage without 
loss of function and thus off-the-shelf availability, and low if any immunogenicity, depending on the phenotype of the parent cell. These considerations underlie Yang's sound proposal $^{1}$ for such a cell-free exosome-based therapy, entailing a protocol whereby a patient's blood cells would first be reprogrammed into induced pluripotent stem cells (iPSCs) before being redifferentiated into cardiomyocytes, from which exosomes could finally be purified.

Some assumptions of the article, though, deserve further discussion. First, Yang ${ }^{1}$ emphasizes the interest of personalizing the therapy through the use of exosomes secreted by autologous iPSC-derived cardiomyocytes. The use of exosomes of a cardiac origin looks sound because (1) even though the exosomal cargo is not a "copy-and-paste" of that of the cytosol of the parent cell but can be selectively enriched, it is highly specific for its seminal phenotype, as illustrated by the failure of fibroblast-derived exosomes to offer any cardioprotection, ${ }^{15}$ and (2) there seems to be a benefit in matching the phenotype of the transplanted cells to that of the target tissue. Possible sources of cardiac cells are limited, however, and, as such, the choice of iPSCs as the exosome producers is attractive, because improvements in their scale up, differentiation, and purification can now be leveraged to derive almost bona fide cardiomyocytes. The use of iPSCs from an autologous source, however, is much more debatable. With accumulating experience, autologous cells have shown their limitations: inconsistent and variable function from one patient to the other, complexity of logistics in the absence of a point-of-care cell processing, and cost of individualized quality controls. The problem is magnified with the use of iPSCs because of the initial requirement for a careful selection of the clones that have been fully reprogrammed, the subsequent check by whole-genome sequencing and methylation profiling that the reprogrammed cells are free from cancer-driving mutations, and the final documentation that the gene expression profile of the redifferentiated cells is consistent with their expected identity (in our case, cardiomyocytes). This is illustrated by the evolution of the Japanese iPSC clinical trial of iPSC-derived retinal pigment epithelial cells in patients with macular degeneration: the study started with autologous iPSCs (it took 1 year to prepare the cells, at a cost of US\$ 1 million for the first patient!) but was stopped at the second patient because of the finding of genetic aberrations ${ }^{16}$; the trial has now been resumed but with the use of allogeneic iPSCs, ${ }^{17}$ allowing the generation of a cell bank, fully qualified for safety and functionality. Cryovials can then be retrieved from the bank when required for a given patient without the need to repeat all the release controls. Obviously, the major drawback of using allogeneic banked cells is their expected elimination by the host's immune system. The risk of this immune rejection could be mitigated, however, by the creation of cell lines homozygous for common HLA phenotypes, allowing selection of the best immunologic match for a large number of potential recipients. ${ }^{18}$ Furthermore, although this issue is critical if cells themselves have to be transplanted, it may not be so relevant if exosomes are to be used because of their reported low immunogenicity, at least when they do not derive from cells of the immune system. Although personalized medicine is increasingly used in oncology because the therapeutic effect of chimeric antigen receptor $\mathrm{T}$ cells is dependent on their ability to target tumor-specific antigens, ${ }^{19}$ this issue is not that relevant to the setting of heart failure. We therefore speculate that to make exosomes "druggable," they will more realistically have to be isolated from banked allogeneic iPSC-derived cardiomyocytes, because it is unlikely that in a period of cost-containment, our societies can afford the cost of autologous iPSC derivatives, at least for treating diseases involving thousands of patients, such as heart failure.

A second important message conveyed by Yang's article is the identification of the miRNA 106a-363 cluster as a key mediator of the beneficial effects of the exosomal cargo of iPSC-derived cardiomyocytes cultured under hypoxic conditions (a setting expected to boost the production of exosomes and also to alter their molecular signature). This assumption is part of a more general strategy whereby attempts have been made to decipher the contents of exosomes and to link some of their components to a given cellular activity, with the hope of identifying a limited number of the most active biologics subsequently amenable to a drug-targeted chemical synthesis. This scientifically appealing approach is nevertheless fraught with limitations. First, there is a wide variability in the identity of the components reported to account for the effects of the exosomal payload. In Yang's article, the key player is the miRNA 106a-363 cluster; in other studies, however, in which exosomes were derived from cardiospheres, a predominant role was attributed to miRNAs $146 a^{12}$ and $148-\mathrm{a}^{20}$ In contrast, miRNA-210 was one of the most enriched in target cells after their uptake of extracellular vesicles from hypoxically cultured human amniotic fluid stem cells, ${ }^{21}$ whereas miRNA $126-3 p$ was the candidate effector of $\mathrm{CD}_{3} 4^{+}$hematopoietic progenitor cell-derived exosomes. ${ }^{22}$ My group's transcriptomic study of the content of extracellular vesicles purified from iPSC-derived cardiovascular progenitors yielded a list of 16 overexpressed miRNAs, not consistently overlapping with those mentioned previously. ${ }^{14}$ These discrepancies can be explained by the fact that the most biologically active components of exosomes likely vary with the cell of origin, the conditions of its culture, and the method used for collecting, purifying, and storing their exosomal cargo. This challenges the generalizability of any reductionist approach. Second, exosomes harbor hundreds, not to say thousands, of proteins and noncoding nucleic acids. Thus, 
although computational models may provide interesting mechanistic insights by predicting the cellular targets of the most strongly expressed miRNAs and proteins in a given extracellular vesicle population, it could be simplistic to conclude that the global protective effect of these particles can then be recapitulated by only a few effectors, in particular because in addition to the intrinsic ability of each to control defined cellular activities, their mutual interactions may also participate in the repair process. A third reason for not trying to deconstruct the exosomal cargo is that a distinct advantage of exosomes is their capacity to be internalized in the target cells and thus to transfer their whole package, protected from degradation by the bilayer lipid coat, more efficiently than if their components were individually delivered (because of enzymatic inactivation or inability of some molecules to cross cell membranes). For all these reasons, keeping the whole content of cell-released exosomes for therapeutic applications might be a better way of leveraging their protective effects.

Finally, Yang postulates that exosome-based therapy for heart failure might undergo a "rapid translation." This optimistic statement should be interpreted cautiously. In contrast to cells (and genes), exosomes are not considered as advanced medicinal therapy products but are classified as biological medications. Their regulation is thus different, which does not mean that it is simpler. It is correct, however, to assume that the greater ease in achieving a consistent quality-controlled manufacturing process than with cells, the availability of in vitro potency assays, and the likely lack of immunogenicity (at least when vesicles are purified from iPSC-derived cardiac-committed cells) might facilitate the approval of exosomes for early phase I trials. The feasibility of such a translation is further supported by the observation that, in oncology, clinical trials with these biologics have already been completed or are ongoing. ${ }^{23}$ This should not lead to underestimating the clinically relevant issues that still need to be addressed. These primarily include the identification of the most efficient "mother" cells (along with cardiac-committed cells, mesenchymal stem cells are extensively investigated as exosome sources because of their high secretory profile), the implementation of a purification method that yields large number of exosomes under sterile conditions without impairing their function, ${ }^{24}$ characterization of the most reliable identity markers, ${ }^{25}$ and optimal dosing and delivery modalities.

Despite these caveats, the commentary by Yang $^{1}$ opens the appealing perspective of leveraging the paracrine effects of stem cells to a "druggable" exosome-based product that duplicates the cells' tissue-repairing capacity. This strategy is fully endorsed by recent data from group's laboratory showing that it may now be worth considering cardiomyocytes derived from pluripotent stem cells (from both embryonic ${ }^{13}$ and $\mathrm{iPSC}^{14}$ sources) no longer as entities only geared for transplantation (with all the safety issues it raises) but rather as ex vivo biofactories generating biologics endowed with equivalent cardioprotective properties. The major clinically relevant advantage of this strategy is that, regardless of whether these biologics would be restricted to exosomes or more broadly encompass other components of the cells' secretome, they would become the only therapeutics given to the patient, thereby bringing stem cells closer to a pharmalike, more user-friendly model.

\section{Conflict of Interest Statement}

Author has nothing to disclose with regard to commercial support.

\section{References}

1. Yang PC. Induced pluripotent stem cell (iPSC)-derived exosomes for precision medicine in heart failure. Circ Res. 2018;122:661-3.

2. Kowal J, Tkach M, Théry C. Biogenesis and secretion of exosomes. Curr Opin Cell Biol. 2014;29:116-25.

3. Vlassov AV, Magdaleno S, Setterquist R, Conrad R. Exosomes: Current knowledge of their composition, biological functions, and diagnostic and therapeutic potentials. Biochim Biophys Acta. 2012;1820:940-8.

4. Pitt JM, Kroemer G, Zitvogel L. Extracellular vesicles: masters of intercellular communication and potential clinical interventions. J Clin Invest. 2016;126: $1139-43$.

5. Cervio E, Barile L, Moccetti T, Vassalli G. Exosomes for intramyocardial intercellular communication. Stem Cells Int. 2015;2015:482171.

6. Tseliou E, Fouad J, Reich H, Slipczuk L, de Couto G, Aminzadeh M, et al. Fibroblasts rendered antifibrotic, antiapoptotic, and angiogenic by priming with cardiosphere-derived extracellular membrane vesicles. J Am Coll Cardiol. 2015;66:599-611.

7. Garbern JC, Lee RT. Cardiac stem cell therapy and the promise of heart regeneration. Cell Stem Cell. 2013;12:689-98.

8. Akyurekli C, Le Y, Richardson RB, Fergusson D, Tay J, Allan DS. A systematic review of preclinical studies on the therapeutic potential of mesenchymal stromal cell-derived microvesicles. Stem Cell Rev. 2015;11:150-60.

9. Adamiak M, Cheng G, Bobis-Wozowicz S, Zhao L, Kedracka-Krok S, Samanta A, et al. Induced pluripotent stem cell (iPSC)-derived extracellular vesicles are safer and more effective for cardiac repair than iPSCs. Circ Res 2018;122:296-309

10. Lai RC, Arslan F, Lee MM, Sze NS, Choo A, Chen TS, et al. Exosome secreted by MSC reduces myocardial ischemia/reperfusion injury. Stem Cell Res. 2010;4: 214-22.

11. Barile L, Milano G, Vassalli G. Beneficial effects of exosomes secreted by cardiac-derived progenitor cells and other cell types in myocardial ischemia. Stem Cell Investig. 2017;4:93.

12. Ibrahim AG-E, Cheng K, Marbán E. Exosomes as critical agents of cardiac regeneration triggered by cell therapy. Stem Cell Reports. 2014;2:606-19.

13. Kervadec A, Bellamy V, El Harane N, Arakélian L, Vanneaux V, Cacciapuoti I, et al. Cardiovascular progenitor-derived extracellular vesicles recapitulate the beneficial effects of their parent cells in the treatment of chronic heart failure J Heart Lung Transplant. 2016;35:795-807.

14. El Harane N, Kervadec A, Bellamy V, Pidial L, Neametalla HJ, Perier MC, et al Acellular therapeutic approach for heart failure: in vitro production of extracellular vesicles from human cardiovascular progenitors. Eur Heart J. February 6, 2018 [Epub ahead of print].

15. Barile L, Lionetti V, Cervio E, Matteucci M, Gherghiceanu M, Popescu LM, et al. Extracellular vesicles from human cardiac progenitor cells inhibit cardiomyocyte apoptosis and improve cardiac function after myocardial infarction. Cardiovasc Res. 2014;103:530-41.

16. Mandai M, Watanabe A, Kurimoto Y, Hirami Y, Morinaga C, Daimon T, et al Autologous induced stem-cell-derived retinal cells for macular degeneration. N Engl J Med. 2017;376:1038-46.

17. Cyranoski D. Japanese man is first to receive "reprogrammed" stem cells from another person. Nature. March 2017. Available at: https://www.nature.com/ 
news/japanese-man-is-first-to-receive-reprogrammed-stem-cells-from-anotherperson-1.21730. Accessed April 30, 2018.

18. Taylor CJ, Peacock S, Chaudhry AN, Bradley JA, Bolton EM. Generating an iPSC bank for HLA-matched tissue transplantation based on known donor and recipient HLA types. Cell Stem Cell. 2012;11:147-52.

19. Xu D, Jin G, Chai D, Zhou X, Gu W, Chong Y, et al. The development of CAR design for tumor CAR-T cell therapy. Oncotarget. 2018;9:13991-4004.

20. Aminzadeh MA, Rogers RG, Fournier M, Tobin RE, Guan X, Childers MK, et al. Exosome-mediated benefits of cell therapy in mouse and human models of Duchenne muscular dystrophy. Stem Cell Reports. 2018;10: 942-55.

21. Balbi C, Piccoli M, Barile L, Papait A, Armirotti A, Principi E, et al. First characterization of human amniotic fluid stem cell extracellular vesicles as a powerful paracrine tool endowed with regenerative potential. Stem Cells Transl Med. 2017;6:1340-55.

22. Mathiyalagan P, Liang Y, Kim D, Misener S, Thorne T, Kamide CE, et al. Angiogenic mechanisms of human $\mathrm{CD} 34^{+}$stem cell exosomes in the repair of ischemic hindlimb. Circ Res. 2017;120:1466-76.

23. Ohno S, Drummen GP, Kuroda M. Focus on extracellular vesicles: development of extracellular vesicle-based therapeutic systems. Int J Mol Sci. 2016;17:172.

24. Heinemann ML, Ilmer M, Silva LP, Hawke DH, Recio A, Vorontsova MA, et al Benchtop isolation and characterization of functional exosomes by sequential filtration. J Chromatogr A. 2014;1371:125-35.

25. Lener T, Gimona M, Aigner L, Börger V, Buzas E, Camussi G, et al. Applying extracellular vesicles based therapeutics in clinical trials-an ISEV position paper. J Extracell Vesicles. 2015;4:30087. 